\title{
Thrombus Size and Doppler Embolic Signal Intensity
}

\author{
Matthew J. Martin ${ }^{a} \quad$ Emma M.L. Chung ${ }^{b}$ Kumar V. Ramnarine ${ }^{a}$ \\ Alison H. Goodall ${ }^{b}$ A. Ross Naylor ${ }^{a}$ b David H. Evans ${ }^{a}$ b \\ a University Hospitals of Leicester NHS Trust and ${ }^{b}$ Department of Cardiovascular Sciences, University of Leicester, \\ Leicester, UK
}

\section{Key Words}

Carotid endarterectomy • Micro-embolic signals •

Thrombus formation · Transcranial Doppler ultrasound •

Embolus detection

\begin{abstract}
Background: Migration of thrombus through the cerebral arteries is a common cause of stroke. Thrombus emboli can be detected non-invasively using Doppler ultrasound, but even where the embolus composition is known, there is currently no method for estimating the size of an embolus based on the returned ultrasound signal. Here we report the results of in vitro experiments investigating the relationship between size and embolic signal intensity for fresh thrombus emboli with a view to estimating the sizes of thrombi detected following carotid surgery. Method: Thrombi were formed from whole blood using the 'Chandler loop' method under flow conditions similar to those associated with arterial thrombus formation in vivo. A total of 390 Doppler embolic signals were then measured from 37 pieces of thrombus circulated in a pulsatile closed-flow circuit. The dimensions of each of the thrombi were measured before and after circulation using an optical microscope. Relationships between thrombus size and embolic signal properties were then investigated using standard statistical methods with a view to size estimation of thrombi during clinical monitor-
\end{abstract}

ing. Results: Although embolic signals generally became more intense with increasing thrombus size, strong oscillations due to resonance effects were observed. Pearson tests revealed strong positive correlations between embolus diameter, signal intensity and duration $(r>0.8, p \leq 0.01)$. Conclusions: This study provides experimental evidence supporting theoretical predictions relating Doppler embolic signal intensity to thrombus size. In our discussion, we tentatively suggest how this information might be used to size emboli in clinical practice.

Copyright $\odot 2009$ S. Karger AG, Basel

\section{Introduction}

Hypercoagulability following surgical trauma can lead to devastating clinical complications and is a common cause of embolic stroke [1]. Fortunately, thrombogenic events can be prevented and averted by appropriate patient monitoring and pharmacological intervention $[2$, 3]. A useful tool for monitoring emboli is transcranial Doppler (TCD) ultrasound [4]. This gives a good indication of particle velocity but is of limited use in determin-

\section{M.J.M. and E.M.L.C. contributed equally to this work.}

\section{KARGER \\ Fax +4161306 1234 E-Mail karger@karger.ch} www.karger.com
(C) 2009 S. Karger AG, Basel 1015-9770/09/0284-0397\$26.00/0 www.karger.com/ced
Prof. David H. Evans

Level 1, Sandringham Building, Leicester Royal Infirmary

Leicester LE1 5WW (UK)

Tel. +44 116258 5610, Fax +44 116258 6070, E-Mail dhe@le.ac.uk 
ing more clinically relevant embolus properties such as embolus size and composition. For example, a small strongly scattering air bubble produces a similar Doppler signal as a large but weakly scattering thrombus. In this study, we investigate the scattering properties of fresh arterial thrombus. The basic premise of our research is that under clinical circumstances where the composition of the particles is known, it may be possible to estimate thrombus size based on prior knowledge of the relationship between thrombus size and Doppler embolic signal intensity.

Since emboli are likely to have differing clinical relevance depending on their size, even a rudimentary estimation of embolus size would provide valuable information to correlate embolus size, composition and prevalence with stroke risk. During the postoperative phase of carotid endarterectomy, embolic signals are thought to be almost entirely due to fresh thrombus emboli formed at the site of the incision [2]. These conditions provide a vital clue to the composition of emboli detected during clinical monitoring, producing a particular instance in which there is potential to form an estimate of embolus size. Thrombus emboli have a density similar to blood and generate weak embolic signals possessing a characteristic distribution of intensities $[5,6]$. Provided emboli are of known composition, comparison with theoretical predictions and in vitro studies may provide a means of placing upper and lower limits on the sizes of clinically observed emboli. This capability could potentially improve the clinical usefulness of Doppler embolus detection.

The relationship between the dimensions of an embolus and its scattering properties depends on the size of the particle in relation to the wavelength of the incident ultrasound beam. If the particle is much smaller than the wavelength, scattering occurs in a predictable isotropic pattern known as Rayleigh scattering. However, for larger particles (with diameters approaching the incident wavelength), the scattering becomes influenced by resonance, interference and other scattering effects and is dependent on angle. This is termed anisotropic or non-Rayleigh scattering.

Theoretical studies have long predicted an oscillatory relationship between particle size and embolic signal intensity due to the effects of resonance phenomena. Calculations performed by Nishi [7] in 1972 predicted that the relationship between the size of a rigid sphere and the intensity of backscattered ultrasound was non-monotonic. Lubbers and van den Berg [8] extended Nishi's work to include deformable spheres, enabling calculation of the theoretical scattering cross-section for red-cell aggregates. In the 1990s, Moehring and Klepper [9] and Moehring and Ritcey [10] published calculations predicting the relationship between embolus diameter and embolus-toblood ratio for solid and gaseous emboli. One limitation is that these calculations assume emboli to be perfectly spherical. Moehring and Klepper [9] speculated that '... sharp resonances are unlikely to occur for more irregularly shaped objects'. Until now, in vitro studies of ultrasonic backscatter from emboli have not measured embolic signal properties with sufficient accuracy to resolve resonance behaviour. In vitro studies, conducted by Russell et al. [11] and Markus and Brown [12] confirmed that larger emboli tend to produce more intense signals, but there were wide variances in these data and the exact relationship remained unclear.

The aim of our in vitro study was therefore to measure the relationship between Doppler embolic signal intensity and size for fresh thrombus. Measurements were performed with sufficient accuracy to allow comparison with theoretical predictions. A tentative framework for estimating embolus sizes during clinical monitoring is suggested.

\section{Method}

Thrombus Preparation

Clinically realistic thrombi were prepared using the Chandler loop method. This involves circulating blood in a closed loop of plastic tubing, which is rotated on a turntable at a speed of $36 \mathrm{rpm}$ for $90 \mathrm{~min}$. A thrombus forms at the forward meniscus of the blood in the tube [13-15]. The resultant thrombi show very similar morphology to human thrombi formed in vivo, including a dense platelet-rich head and fibrin-rich tail [15]. Freshly prepared thrombi were cut into small pieces between 0.3 and $2.3 \mathrm{~mm}$ in diameter, measured using a travelling optical microscope equipped with a Vernier scale capable of $\pm 0.01 \mathrm{~mm}$ accuracy. Mean embolus diameter was estimated based on an average of 3 orthogonal measurements. This method of sizing is at least as accurate as measurement techniques employed in previous in vitro studies $[11,12]$.

Ethical permission for this study was granted by our regional research ethics committee.

\section{Flow Rig Experiments}

Flow rig experiments were performed using a closed-loop flow phantom (fig. 1a). The circuit was constructed from C-flex tubing with $4.6 \mathrm{~mm}$ internal diameter and $0.8 \mathrm{~mm}$ wall thickness (ColeParmer, London, UK). C-flex tubing has a density of $886 \mathrm{~kg} \cdot \mathrm{m}^{-3}$, acoustic velocity of $1,553 \mathrm{~m} \cdot \mathrm{s}^{-1}$ and attenuation of $28 \mathrm{~dB} \cdot \mathrm{cm}^{-1}$. $\mathrm{MHz}^{-1}$, compared to $1,000 \mathrm{~kg} \cdot \mathrm{m}^{-3}, 1,540 \mathrm{~m} \cdot \mathrm{s}^{-1}$ and $0.5 \mathrm{~dB} \cdot \mathrm{cm}^{-1}$. $\mathrm{MHz}^{-1}$ for tissue [16]. This is an identical experimental arrangement to that described in a previous publication [17]. 


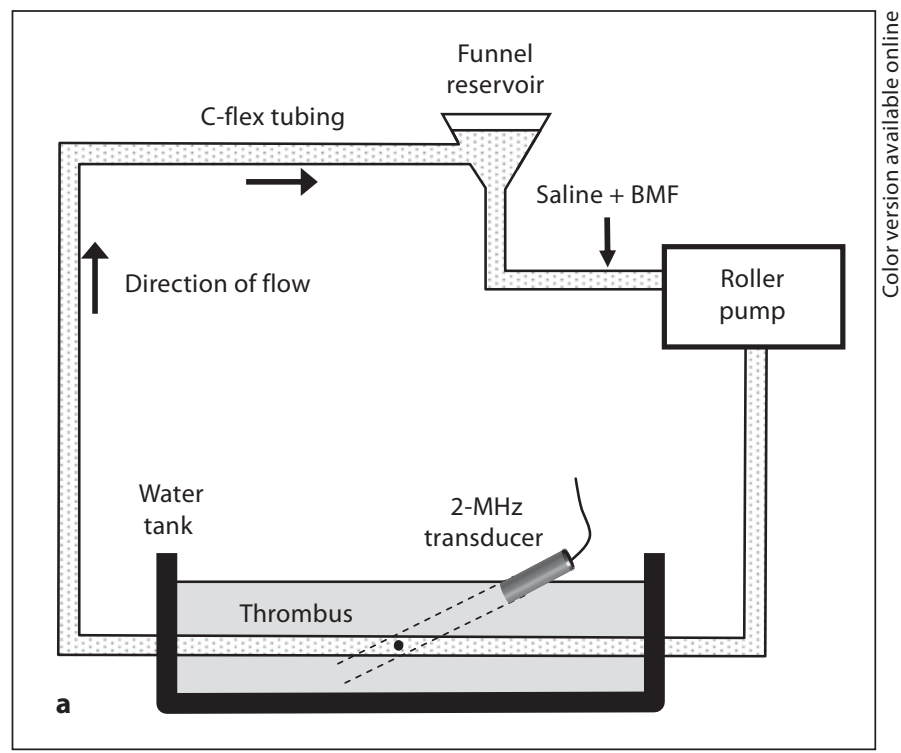

Fig. 1. a Closed-loop flow rig designed to evaluate the mean embolic signal intensity of circulating thrombi. The flow circuit was filled with $0.9 \%$ saline plus $0.2 \%$ orgasol (BMF = blood mimicking fluid) particles. Pulsatile flow peaking at approximately $30 \mathrm{~cm} \cdot \mathrm{s}^{-1}$ was generated using a peristaltic pump. b Analysis of embolic signals was performed using a Graphical User Interface programmed in Matlab. An embolus moving through the Doppler sample volume produces an increase in backscattered ultrasound. This was measured relative to the background signal from surrounding blood flow. Embolus and background 'windows' were chosen manually (shown as the dashed lines).

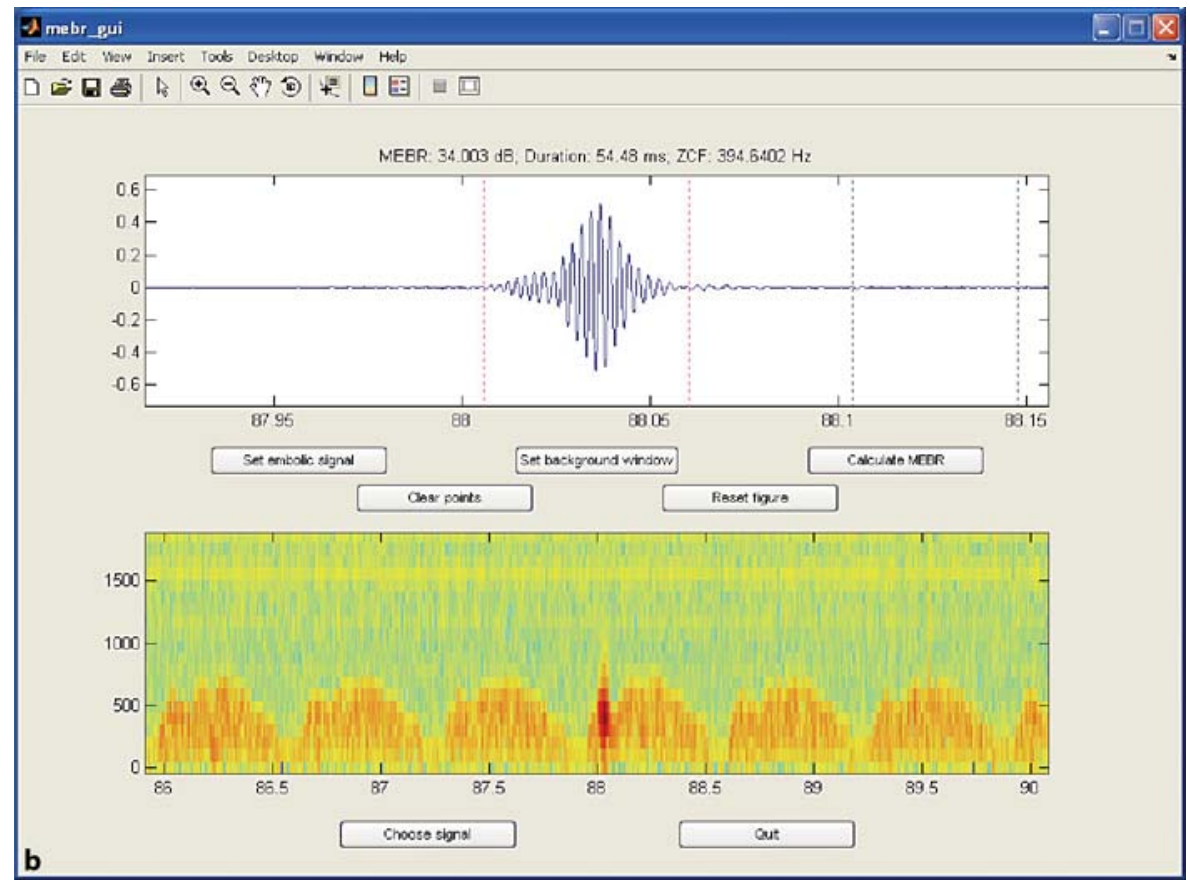

Pulsatile flow, with a peak systolic velocity of $30 \mathrm{~cm} \cdot \mathrm{s}^{-1}$, was generated using a peristaltic pump (HR Flow Inducer Type 2000, Watson Marlow, Falmouth, UK). The circulating fluid consisted of $100 \mathrm{ml}$ of $0.9 \%$ saline mixed with $0.2 \%$ orgasol scatterers. This dilute suspension of orgasol scatterers generated a Doppler background signal that was approximately $1 / 50$ th the strength of blood [18]. This enabled us to use a transparent fluid, while still obtaining an easily measurable background Doppler signal. In later calculations we compensated for dilution of the blood mimic by scaling the background intensity by a factor of 50 . The fluid was circulated to remove air from the system and a single piece of thrombus of known size introduced via the funnel reservoir. Each piece of thrombus circulated past the transducer at least 10 times over a period of $<10$ min generating several measurements of embolic signal properties per thrombus. Optical microscopy was performed before and after circulation to confirm that no loss of thrombus volume had occurred. All of the thrombi were freshly made and were kept moist to avoid shrinkage due to dehydration. Data from thrombi that had fragmented on passing through the pump were discarded.

Since the circulating fluid was transparent, passage of an embolus through the sample volume could be verified by visual in- 
Fig. 2. a The relationship between average MEBR and mean embolus diameter followed an oscillatory behaviour. Error bars indicate the standard deviation. The greyscale image shows the distribution of underlying data points (diameter bin width of $0.1 \mathrm{~mm}$ and an MEBR bin width of $2 \mathrm{~dB}$ ). Note that the curve has been scaled (via equation 1) to provide an estimate of approximate relative embolic signal intensity relative to full-strength blood mimic. b Theoretical prediction for the backscatter cross-section of red-cell aggregates in saline. The theoretical result has been normalized so that the minimum value of the curve is $0 \mathrm{~dB}$.

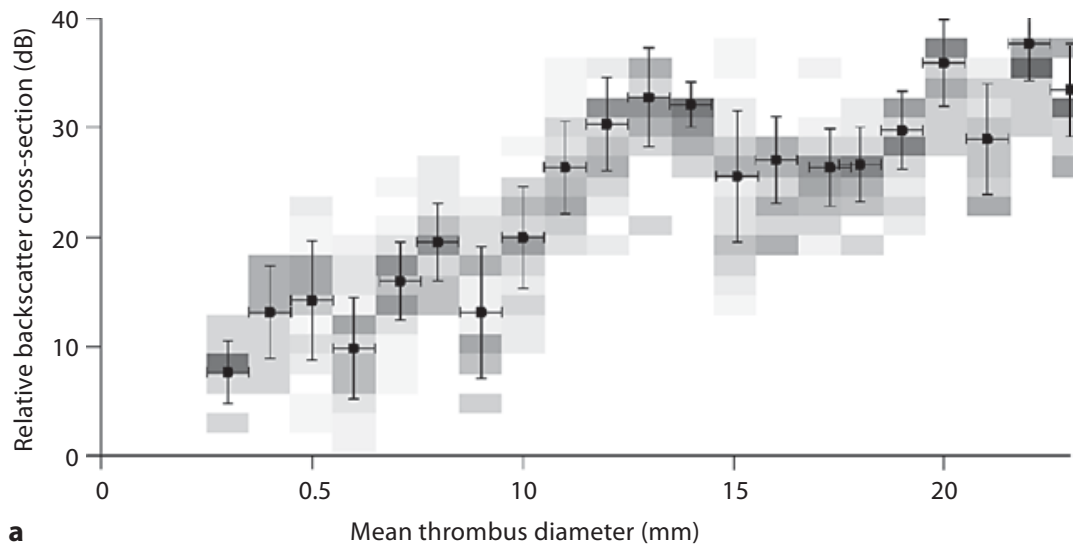

a

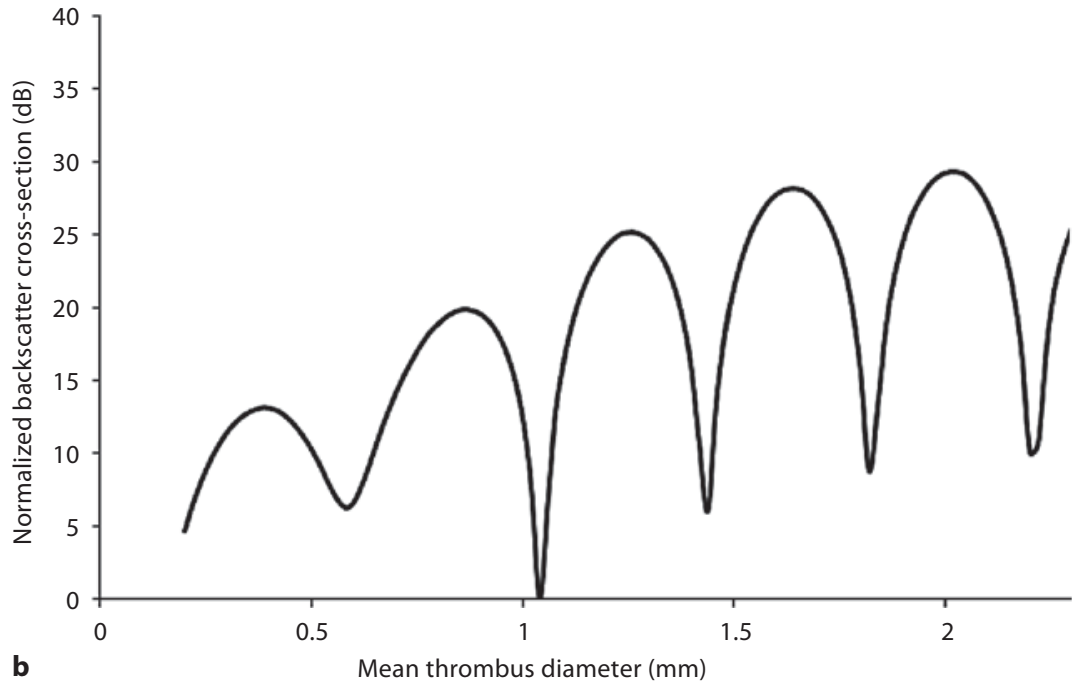

spection. The smallest particle resolvable by eye was $0.3 \mathrm{~mm}$ in diameter, which limited the observable size range between 0.3 and $2.3 \mathrm{~mm}$. The upper limit of $2.3 \mathrm{~mm}$ is slightly less than the diameter of the middle cerebral artery. Embolic signals were accepted for analysis only if the time of appearance of the embolic signal in the sonogram matched the passage of the thrombus through the sample volume (visually recorded by an observer). All of the accepted embolic events had properties that were consistent with international embolus detection criteria [19].

The Doppler system used in this study was a non-commercial multi-gate TCD device with high dynamic range to avoid saturation of the signal at high measured embolus-to-background ratio (MEBR) values [20]. This device made it possible to extract the raw audio data for further analysis in Matlab (Mathworks Inc., Natick, Mass., USA). TCD system settings were compared to those of a commercial TC22 TCD machine (Scimed, Bristol, UK) and found to have lower thermal index, lower pulse-pressure amplitude and similar signal-to-noise ratio [21]. To mimic insonation of the middle cerebral artery, the artificial vessel was insonated using a nominal $10-\mathrm{mm}$ sample length, at a depth of $50 \mathrm{~mm}$ and insonation angle of $30^{\circ}$. Data recorded using this equipment were saved directly to hard disk for later analysis.

Analysis of embolic signal data was performed using a Graphical User Interface programmed in Matlab (fig. 1b). Embolus and background windows were selected by the operator to ensure that there were no artefacts present in either of the windows (fig. 1b). Each window was then integrated and normalized with respect to time to calculate the approximate relative embolic signal intensity in terms of a MEBR via equation 1 [4]:

$$
M E B R=10 \log _{10}\left(\frac{E+50 B}{50 B}\right) \mathrm{dB}
$$

where $E$ and $B$ are the integrated embolus and background audio signals normalized with respect to time. Note that the measured background signal is scaled by a factor of 50 to compensate for the concentration of background scatterers in the circulating fluid being $1 / 50$ th of that of blood. This is based on the assumption that at low concentrations the relationship between backscatter power and number of scatterers is approximately linear [18]. Other prop- 
erties extracted from the Doppler audio signal included the duration of the embolic signal in milliseconds (measured by the user), power of the embolus and background signals, and zero-crossing frequency in hertz. The zero-crossing frequency was then used to calculate embolus velocity via the Doppler equation. Velocities were angle-corrected based on a Doppler angle of $30^{\circ}$.

\section{Statistical Analysis}

Mean MEBR (decibels), signal duration (milliseconds), signal frequency (hertz) and associated standard deviations were estimated for each thrombus from an average of at least 10 measurements. Correlations between mean embolus diameter and embolic signal properties such as MEBR, signal duration and velocity were evaluated using a standard Pearson correlation test to a level of significance of $\mathrm{p} \leq 0.01$.

\section{Results}

A total of 390 measurements were obtained from 37 thrombi with diameters ranging between 0.3 and 2.3 $\mathrm{mm}$. A mean MEBR value was estimated for each thrombus from a minimum of 10 circulations through the Doppler sample volume. The measured relationship between average thrombus diameter in millimetres and mean MEBR in decibels for each of the particles is plotted alongside the theoretical prediction for the backscatter cross-section of red-cell aggregates in saline (normalized to a minimum of $0 \mathrm{~dB}$; fig. 2). The theoretical graph has been calculated using the model developed by Lubbers and van den Berg [8]. Physical properties assumed for saline and red-cell aggregates are listed in table 1 . The greyscale image and y-error bars in the upper panel indicate the spread of the experimental data points. Circulation of each thrombus under pulsatile flow conditions produced a range of MEBR values with a standard deviation of $\pm 5 \mathrm{~dB}$.

Our in vitro measurements clearly confirm the presence of resonance effects. Average signal intensity, duration and embolus velocity for each thrombus size are shown in figure $3 \mathrm{a}-\mathrm{c}$. The radius of the markers is proportional to thrombus diameter. Trend lines are indicated by the dashed lines. Figure $3 \mathrm{~d}$ shows a strong positive correlation between MEBR and duration. For signals with similar intensities, larger emboli were found (on average) to generate signals of longer duration than smaller emboli with the same MEBR. In figure 3e, an inverse relationship between velocity and duration can be observed.

Statistical analyses were performed for the 390 individual measurements (table 2, left) and for data that had been grouped by thrombus size to the nearest $0.1 \mathrm{~mm}$
Table 1. Theoretical assumptions for modelling the relationship between thrombus diameter and embolic signal intensity measured using Doppler ultrasound

\begin{tabular}{lll}
\hline Model assumptions & $\begin{array}{l}\text { Density } \\
\mathrm{kg} \cdot \mathrm{m}^{-3}\end{array}$ & $\begin{array}{l}\text { Sound velocity } \\
\mathrm{m} \cdot \mathrm{s}^{-1}\end{array}$ \\
\hline Whole blood $\left(37^{\circ} \mathrm{C}\right)$ & $1,055[9]$ & $1,580[9]$ \\
Normal saline $\left(20^{\circ} \mathrm{C}\right)$ & $1,005[28]$ & $1,491[29]$ \\
BMF & $1,037[16]$ & $1,548[16]$ \\
Red cell aggregates & $1,090[9]$ & $1,640[9]$ \\
Plasma & $1,030[9]$ & $1,542[9]$
\end{tabular}

Assumptions for 'physiologically realistic' values of MEBR

\begin{tabular}{lll}
\hline Blood & hematocrit & $45 \%$ \\
Vessel & diameter & $3 \mathrm{~mm}$ \\
& SVL & $10 \mathrm{~mm}$ \\
Ultrasound & frequency & $2 \mathrm{MHz}$
\end{tabular}

$\mathrm{BMF}=$ Blood mimicking fluid; SVL = sample volume length.

Table 2. Pearson tests for correlations between pairs of embolic signal parameters and mean thrombus diameter

\begin{tabular}{|c|c|c|c|c|c|c|}
\hline & \multicolumn{3}{|c|}{$\begin{array}{l}\text { Individual raw data } \\
\text { points ( } 370 \text { signals) }\end{array}$} & \multicolumn{3}{|c|}{$\begin{array}{l}\text { Grouped averaged data } \\
\text { (21 thrombus sizes) }\end{array}$} \\
\hline & MEBR & velocity & duration & MEBR & velocity & duration \\
\hline Size & $0.76^{1}$ & -0.11 & 0.48 & $0.89^{1}$ & -0.63 & 0.9 \\
\hline Duration & 0.5 & -0.21 & & 0.84 & -0.64 & \\
\hline Velocity & -0.5 & & & -0.7 & & \\
\hline
\end{tabular}

The left-hand side of the table shows data for individual raw data points, while the right-hand side shows correlations for data that have been grouped and averaged for each thrombus size. All correlations were measured to a level of significance of $\mathrm{p} \leq 0.01$.

${ }^{1}$ Non-linear relationship (see fig. 2).

(table 2, right). Pearson correlation tests revealed a strong positive correlation between mean embolus diameter and MEBR, a moderate positive correlation between size and signal duration, and a weak negative correlation between embolus size and velocity. By reducing the measurement uncertainty by averaging over multiple measurements, all correlations were strengthened, approaching $\mathrm{r} \approx 0.9$ for correlations between MEBR, size and duration (table 2, right). The presence of a strong average negative correlation $\mathrm{r} \approx 0.7$ between velocity and size/MEBR was also confirmed. 


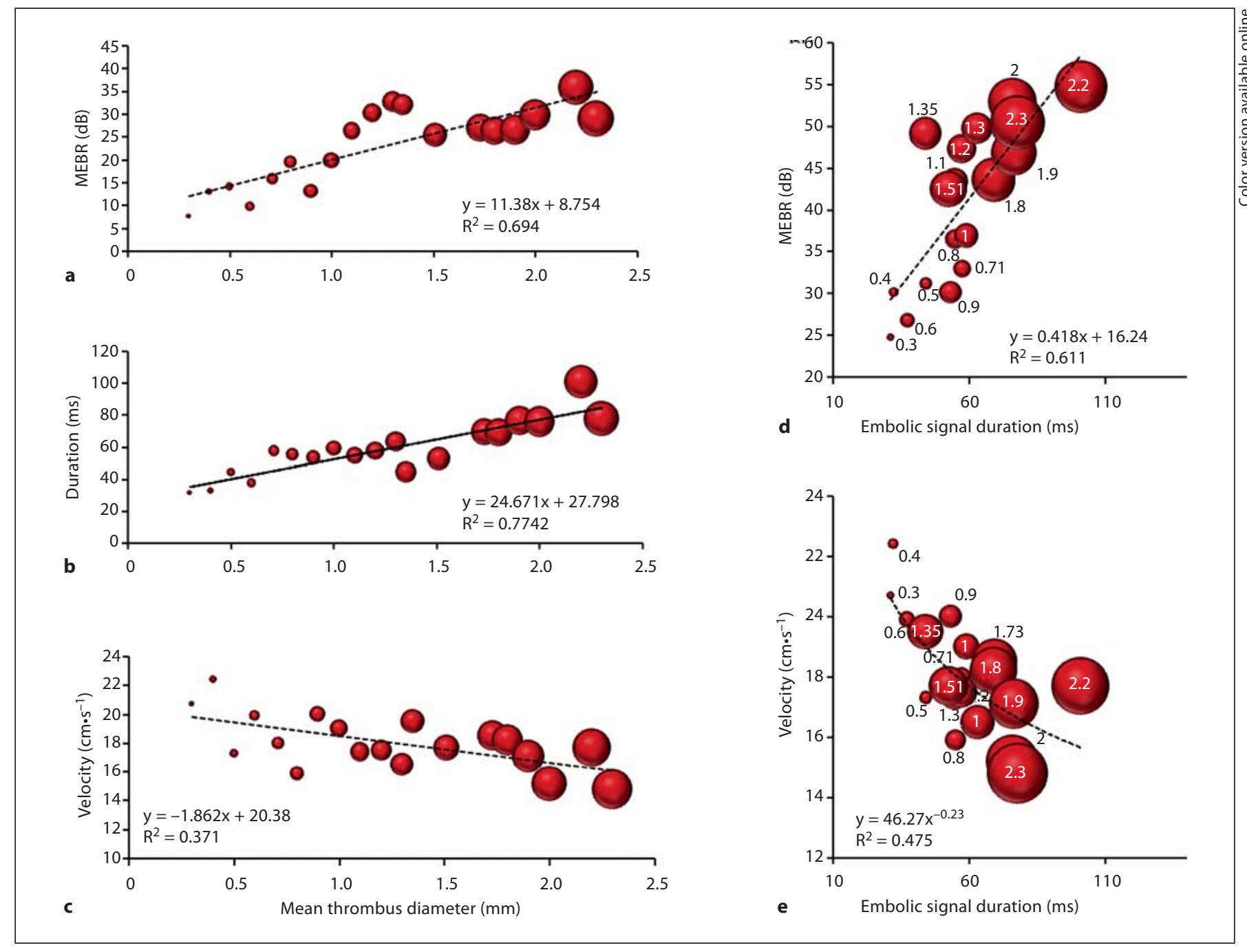

Fig. 3. Variations in estimated mean embolic signal intensity (MEBR) (a), estimated embolic signal duration (b) and embolus velocity (c) with thrombus diameter. In addition to a positive correlation between MEBR and thrombus size ( $\mathrm{r}=0.89, \mathrm{p} \leq 0.01)$, a positive correlation between embolus size and average signal duration was observed $(\mathrm{r}=0.84, \mathrm{p} \leq 0.01)$. A negative correlation between embolus size and velocity $(\mathrm{r}=-0.63, \mathrm{p} \leq 0.01)$ suggests that larger emboli tended to move more slowly than smaller emboli of the same composition. $\mathbf{d}$ There was a strong positive correlation between MEBR and signal duration $(r=0.9, p \leq 0.01)$. e There was a negative correlation between embolus duration and velocity $(\mathrm{r}=-0.64, \mathrm{p} \leq 0.01)$. The diameters of the emboli are indicated by the width of the markers. In $\mathbf{d}$ and $\mathbf{e}$, grouped average thrombus diameters are individually labelled.

\section{Discussion}

Our results provide experimental evidence supporting theoretical predictions of an oscillatory relationship between embolus diameter and Doppler embolic signal intensity. Each thrombus was measured before and after introduction to the phantom to confirm that there was no fragmentation over successive circulations. Our in vitro study featured 390 measurements for thrombi between 0.3 and $2.3 \mathrm{~mm}$ (fig. 2a). For thrombi less than 0.3 $\mathrm{mm}$ in diameter, the relationship between MEBR and thrombus size is expected to tend to zero without further oscillations. Further studies will be required to examine the quantitative predictive capacity of size estimation and to assess whether a rudimentary size estimate could be refined to provide useful information in clinical practice.

Size estimation is likely to be hindered by variations in MEBR that are intrinsic to the measurement process. These arise from a number of factors including non-uni- 
form insonation of the sample volume, variations in background scattering and anisotropic (non-Rayleigh) scattering. Physiologically realistic emboli are also likely to be unevenly shaped due to irregular formation of pathological material and the random nature of detachment of pieces of thrombus as emboli. Irregular emboli with dimensions similar to the wavelength of incident ultrasound are unlikely to scatter ultrasound in a predictable manner. A further limitation of our study is the examination of a single composition of embolus. We stress that results relating to fresh thrombus would not apply to other types of less homogeneous emboli containing cholesterol or plaque components.

During in vitro experiments, distortion of the beam by the walls of the C-flex tubing and differences in the flow pattern (which in our study was pulsatile but nonphysiological) may also have affected MEBR estimates. If the lumen of the artery is not uniformly insonated, MEBR values will be strongly dependent on the trajectory of the embolus as it passes through the sample volume [22, 23]. Distortion of the ultrasound beam by the skull and tissue inhomogeneities is expected to affect MEBR values by approximately $4 \mathrm{~dB}$ [22]. Ultrasound techniques are currently being developed to help overcome this problem [24] and are expected to permit more accurate clinical measurements of embolic signal properties in the future.

\section{How Could Embolus Sizing Be Applied to}

Interpretation of Clinical Embolus Detection Data?

If the composition of emboli can be deduced from consideration of the clinical circumstances, this would theoretically facilitate embolus sizing. As a tentative exploration of whether it would be feasible to place limits on the upper diameters and size ranges of emboli detected during clinical monitoring, we applied theoretical predictions for red-cell aggregates in blood to analysis of the distribution of embolic signal intensities. Previous studies suggest that patients who are micro-embolic signal positive following carotid surgery are approximately 15 times more likely to experience a stroke or transient ischaemic attack [25]. As postoperative embolization is thought to be caused predominantly by formation of fresh thrombus at the site of the incision [2], it seems reasonable to assume that emboli observed following carotid endarterectomy are likely to consist of fresh arterial thrombus.

To explore the clinical feasibility of embolus sizing we analysed an ultrasound recording obtained from a 67year-old female patient with 751 embolic signals detected

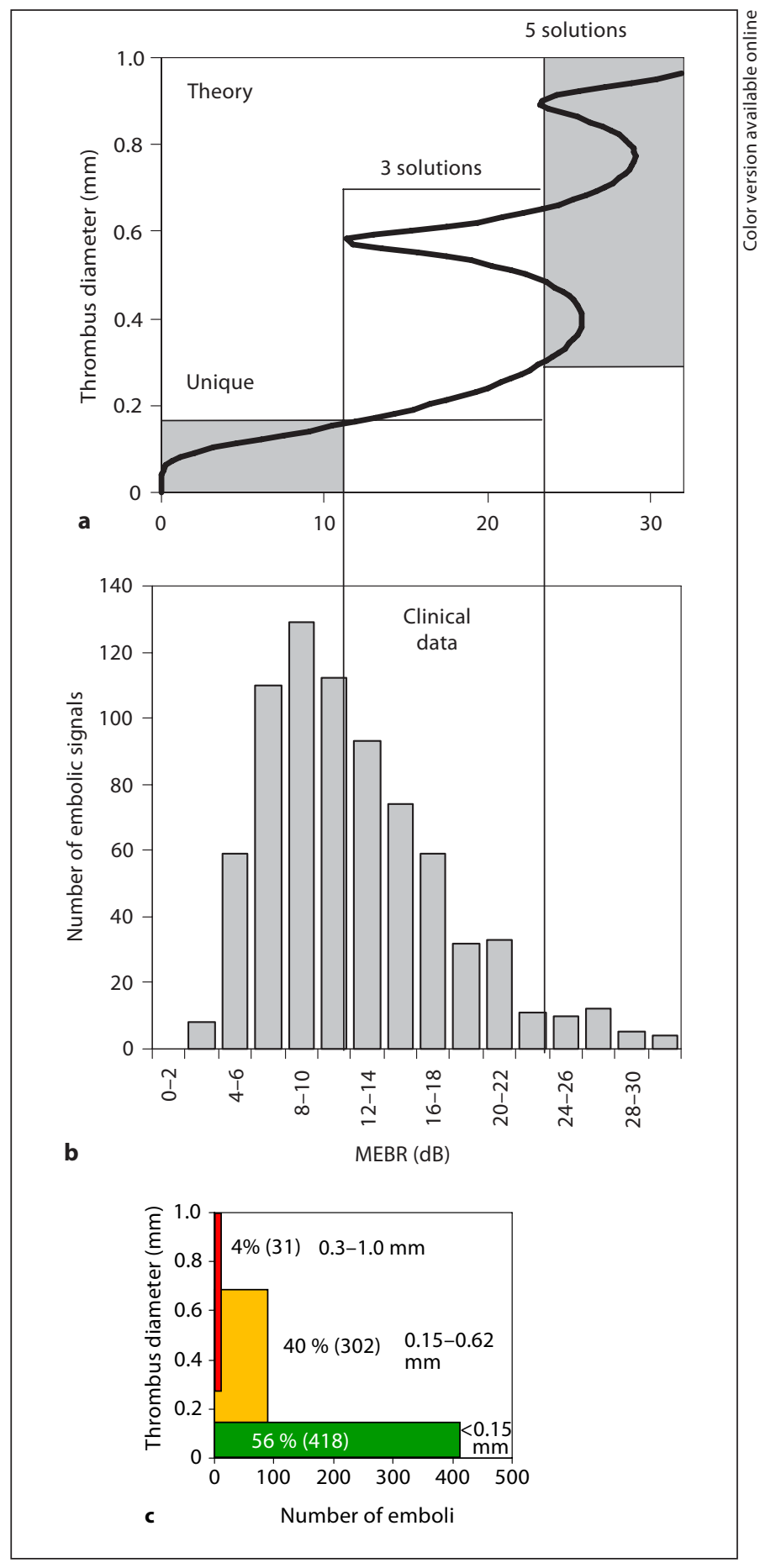

Fig. 4. Distribution of embolic signal intensities for a patient with high-grade embolization following carotid surgery (b). The theoretical curve (a) has been calculated using the values for blood, plasma and red-cell aggregates provided in table 1. Assuming that theoretical predictions for thrombus emboli in blood can be applied in vivo, the majority of signals are less than $12 \mathrm{~dB}$ in intensity (a). This corresponds to thrombus diameters less than 0.15 $\mathrm{mm}$ (c). For emboli producing intensities $>12 \mathrm{~dB}$, multiple discrete sizes of emboli are consistent with each intensity. 
after carotid endarterectomy. The patient had experienced a transient ischaemic attack 1 week prior to surgery resulting in left-sided weakness and dysphasia. On angiography she was found to have a total left carotid occlusion and $90 \%$ occlusion of the right carotid artery. She underwent an emergency right-side carotid endarterectomy during which the intra-operative rate of embolization was unremarkable. Following surgery we performed $5 \mathrm{~h}$ of postoperative monitoring and recorded a total of 751 embolic signals. These were manually analysed offline to determine the distribution of embolic signal intensities. Data collection and analysis of the recordings were performed using identical software and embolus detection criteria as used for analysis of the in vitro data. The intensity distribution of embolic signals detected during postoperative clinical monitoring closely resembled previous reports $[5,6]$. Despite experiencing a high number of emboli postoperatively, the patient made a full recovery from the operation and experienced no obvious neurological deficits.

Comparison with theoretical MEBR predictions for thrombus circulating in blood, calculated based on the assumptions for blood, plasma and red-cell aggregates cited in table 1 , suggests that $56 \%$ (418/751) of emboli possessed a uniquely determined range of sizes of less than $0.15 \mathrm{~mm}$ in diameter (fig. 4a). Above approximately 12 $\mathrm{dB}$, we find that multiple sizes of emboli are consistent with each MEBR value (i.e. there are multiple discrete solutions). In this regime approximately $40 \%$ (302) of embolic signals had intensities between 12 and $24 \mathrm{~dB}$, placing an upper limit on embolus size of $0.62 \mathrm{~mm}$ diameter and a lower limit of $0.15 \mathrm{~mm}$. Approximately $4 \%$ (31) of the emboli produced strong signals between 24 and 32 $\mathrm{dB}$. This would theoretically correspond to a size range between 0.3 and $1 \mathrm{~mm}$ in diameter.

The patient experienced over 700 emboli with no obvious neurological deficits. We therefore assume that the majority of fresh arterial thrombus emboli are asymp- tomatic due to their small size and fast clearance time. All of the detected emboli were predicted to be less than $1 \mathrm{~mm}$ in diameter, with the majority (56\%) being uniquely defined with diameters of $0.15 \mathrm{~mm}$ or less. Fresh thrombus formed after surgery often consists of loosely packed platelets that are weakly activated and not stabilized by fibrin $[26,27]$. In this situation embolus size rather than prevalence is likely to be an important factor for quantifying embolic burden. In future studies it might be interesting to determine whether inclusion of additional information combining embolization rate and estimated embolus size would provide an improved marker for stroke risk.

\section{Conclusions}

Our in vitro results for fresh thrombi provide experimental confirmation of the oscillatory nature of the theoretical relationship between thrombus size and MEBR. Exploratory application of theoretical predictions to the interpretation of clinical embolic signals was capable of broadly categorizing thrombi into 3 size ranges: (i) less than $0.15 \mathrm{~mm}$ (56\%), (ii) between 0.15 and $0.62 \mathrm{~mm}$ (40\%) and (iii) between 0.3 and $1 \mathrm{~mm}(4 \%)$. However, further work will be required to improve embolus characterization and to determine which embolic signal properties (if any) can provide an improved marker for stroke risk during clinical monitoring.

\section{Acknowledgement}

This research was supported by the British Heart Foundation and the Wellcome Trust. We thank Sergio Matos and David Buckle for helping to develop the embolic signal analysis GUI. The Doppler system used in this study was developed previously as part of the Ultrasonic Monitoring and Early Diagnosis of Stroke FP7 project.

\section{References}

1 Selim M: Current concepts: perioperative stroke. N Engl J Med 2007;356:706-713.

2 Payne DA, Jones CI, Hayes PD, Thompson MM, London NJ, Bell PR, Goodall AH, Naylor AR: Beneficial effects of clopidogrel combined with aspirin in reducing cerebral emboli in patients undergoing carotid endarterectomy. Circulation 2004;109:14761481.
-3 Beldi G, Beng L, Siegel G, Bisch-Knaden S, Candinas D: Prevention of perioperative thromboembolism in patients with atrial fibrillation. Br J Surg 2007;94:1351-1355.

4 Evans DH: Detection of microemboli; in Babikian VL, Wechsler LR (eds): Transcranial Doppler Ultrasonography, ed 2. Boston, Butterworth Heinemann, 1999, pp 141-155.
5 Chung EML, Fan L, Naylor AR, Evans DH: Characteristics of Doppler embolic signals observed following carotid endarterectomy. Ultrasound Med Biol 2006;32:1011-1023.

-6 Chung EML, Fan L, Naylor AR, Evans DH: Impact of clinical environment on embolus detection: a comparison of automated and manual detection of embolic signals. Cerebrovasc Dis 2007;23:169-174. 
7 Nishi RY: Ultrasonic detection of bubbles with Doppler flow transducers. Ultrasonics 1972;10:173-179.

8 Lubbers J, Van den Berg JW: An ultrasonic detector for microgas emboli in a bloodflow line. Ultrasound Med Biol 1977;2:301-310.

-9 Moehring MA, Klepper JR: Pulse Doppler ultrasound detection, characterization and size estimation of emboli in flowing blood. IEEE Trans Biomed Eng 1994;41:35-44.

-10 Moehring MA, Ritcey JA: Sizing emboli in blood using pulse Doppler ultrasound. I. Verification of the EBR model. IEEE Trans Biomed Eng 1996;43:572-580.

11 Russell D, Brucher R, Madden K, Clark WM, Sandset PM, Zivin J: The intensity of the Doppler signal caused by arterial emboli depends on embolus size; in Oka M, von Teutern GM, Furuhata H, Kodaira K (eds): Recent Advances in Neurosonology. Amsterdam, Elsevier Science Publishing, 1992, pp 57-60.

12 Markus HS, Brown MM: Differentiation between different pathological cerebral embolic materials using transcranial Doppler in an in vitro model. Stroke 1993;24:1-5.

$\checkmark 13$ Chandler AB: In vitro thrombotic coagulation of the blood: a method for producing a thrombus. Lab Invest 1958;7:110-114.

14 Poole JCF: A study of artificial thrombi produced by a modification of Chandler's method. Q J Exp Physiol 1959;44:377-384.
15 Robbie LA, Young SP, Bennett B, Booth NA: Thrombi formed in the Chandler loop mimic human arterial thrombi in structure and PAI-1 content and distribution. Thromb Haemost 1997;77:510-515.

16 Hoskins PR, Ramnarine KV: Doppler test devices; in Evans DH, McDicken WN (eds): Doppler Ultrasound: Principles, Instrumentation and Signal Processing, ed 2. Chichester, Wiley, 2000, pp 382-404.

17 Martin MJ, Chung EML, Goodall AH, Della Martina A, Ramnarine KV, Fan L, Hainsworth SV, Naylor AR, Evans DH: Enhanced detection of thrombo-emboli using targeted microbubbles. Stroke 2007;38:2726-2732.

18 Ramnarine KV, Nassiri DK, Hoskins PR, Lubbers J: Validation of a new blood-mimicking fluid for use in Doppler flow test objects. Ultrasound Med Biol 1998;24:451459.

19 Ringelstein EB, Droste DW, Babikian VL, Evans DH, Grosset DG, Kaps M, Markus HS, Russel D, Siebler M: Consensus on microembolus detection by TCD. Stroke 1998;29:725729.

20 Fan L, Boni E, Tortoli P, Evans DH: Multigate transcranial Doppler ultrasound system with real-time embolic signal identification and archival. IEEE Trans Ultrason Ferroelectr Freq Control 2006;53:1853-1861.

21 Fan L, Ramnarine KV, Gittins J, Evans DH: Performance analyses of two transcranial Doppler systems using an electronic phantom and a flow phantom. Ultrasound 2006; $14: 272$.
22 Angell EL, Evans DH: Limits of uncertainty in measured values of embolus-to-blood ratio due to Doppler sample volume shape and location. Ultrasound Med Biol 2003;29: 1037-1044.

23 Evans DH, Gittins J: Limits of uncertainty in measured values of embolus-to-blood ratios in dual-frequency TCD recordings due to nonidentical sample volume shapes. Ultrasound Med Biol 2005;31:233-242.

24 Aubry JF, Tanter M, Pernot M, Thomas JL, Fink M: Experimental demonstration of noninvasive transskull adaptive focusing based on prior computed tomography scans. J Acoust Soc Am 2003;113:84-93.

25 Abbott AL, Levi CR, Stork JL, Donnan GA, Chambers BR: Timing of clinically significant microembolism after carotid endarterectomy. Cerebrovasc Dis 2007;23:362-367.

26 Oude Egbrinke MGA, van Gestel MA, Boeders MAW, et al: Regulation of microvascular thromboembolism in vivo. Microcirculation 2005;12:287-300

27 Van Gestel MA, Heemskerk JWM, Slaaf DW, et al: Real-time detection of activation patterns in individual platelets during thrombo-embolism in vivo: differences between thrombus growth and embolus formation. J Vasc Res 2002;39:534-543.

28 Kaye GWC, Laby TH: Tables of Physical and Chemical Constants, ed 15. New York, Longman, 1986, p 223.

29 Baker AC, Duck FA, Starritt HC (eds): Ultrasound in Medicine. Boca Raton, CRC Press, 1998. 